\title{
THE MANAGEMENT OF ELECTRICAL BURNS
}

\author{
By I. F. K. MuIR, M.B.E., M.B., F.R.C.S. \\ Mount Vernon Centre for Plastic Surgery
}

Injuries due to electrical currents-so called electrical burns--form a small but important section of all burning accidents. They have certain features which distinguish them from thermal burns, and which have an important bearing on prognosis and treatment.

The majority of electrical burns seen in this country are caused by accidents involving the domestic electrical supply (usually 220-250 volts A.C.), but burns caused by the high voltage grid system (e.g. 66,000 volts, or 33,000 volts) are occasionally seen. These high tension injuries are usually much more severe than those caused by the domestic supply, and are more often fatal.

\section{Physics}

The damage is due to the passage of electricity through the tissues, and the extent and severity of the damage depends on the strength of the current (in amperes) and the time for which contact is maintained.

The voltage is dependent on the nature of the supply, and as has been stated is usually 220-250 volts. The amperage depends upon the resisstance offered to the current by the body in accordance with Ohm's Law.

$$
\mathrm{A}-\frac{\mathrm{V}}{\mathrm{R}}
$$

The large fluid content of tissues, particularly of the blood plasma and interstitual fluids, makes the interior of the body an excellent conductor (i.e. of low resistance), and the main variability of resistance lies in the skin and in the nature of its contact with the source of electricity. Thus wet skin in firm contact with the source will allow more current to pass than dry skin in light contact. It should also be remembered that if current is to pass in the body two contacts must be made. The ordinary two-point domestic supply consists of a live or active wire and a neutral wire. The extra point in a three-point supply is used to earth the frame of the apparatus. Current will pass when one contact is made with the live wire and a second contact with either the neutral wire or the ' earth.' 'The ' earth ' connection may be by actual direct contact with the ground or by indirect contact

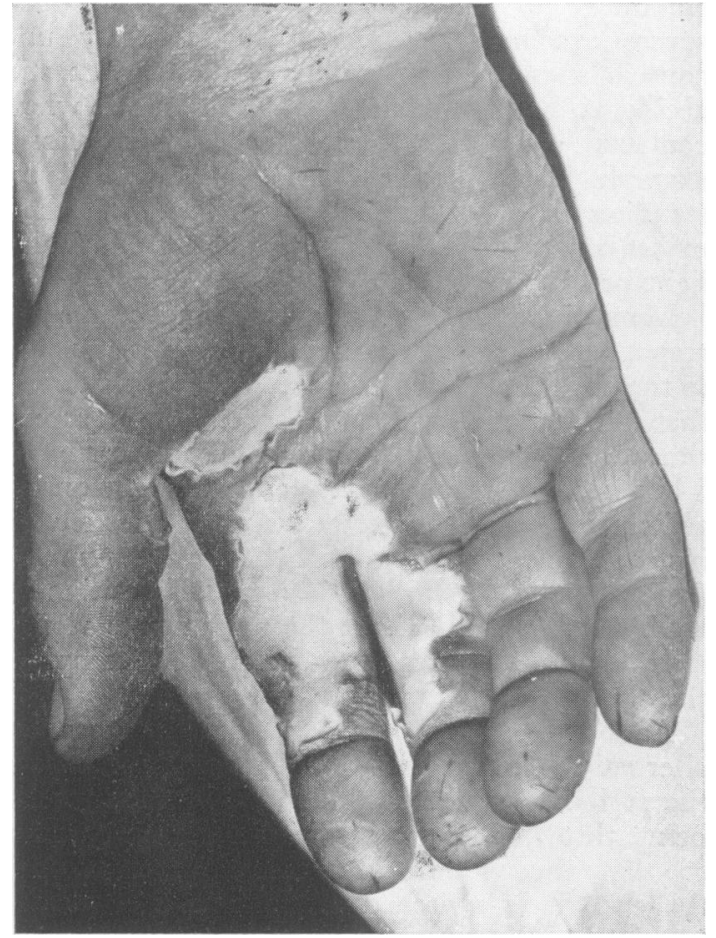

FIg. I.-An electrical burn of the hand. Shows the entry and exit wounds on the same hand. Loose horny skin has been removed. A patient of Mr. Rainsford Mowlem, F.R.C.S.

with a water-pipe or drainpipe. Under ordinary conditions the floor of a house is insulated from the ground and a burn will not occur even when the live wire is firmly held unless this second contact is made.

It will therefore be clear that there will always be an entry and an exit wound although, because of differences in contacts and local resistance, these may differ very much in severity. Often both wounds are on the same hand. Sometimes there is one on each hand, less frequently other parts of the body are involved (Fig. 2).

The hands are involved in the vast majority of accidents. The last 27 patients with domestic 
supply electrical burns admitted to the Mount Vernon Plastic Centre, had burns of the hands, no other part of the body being involved, and of seven patients with high tension injuries, four had severe injuries of one or both hands, two had severe injuries of one arm and only one had no burn on the upper limbs. In all these high tension cases there were severe burns on other parts of the body.

Fortunately the body resistance usually keeps the amount of current below that necessary to produce severe systemic disturbances. Temporary unconsciousness is not uncommon in domestic accidents, but death is rare and only occurs when contacts are exceptionally good, the classic example being the man excellently earthed by standing in a bath filled with water who then makes contact with the mains, by reason of a faulty light or heater switch.

Many different types of electrical apparatus are responsible for accidents: sometimes this is due to the development of a defect in the insulation so that a frayed wire causes a short circuit but sometimes it is due to faulty construction or assembly. Thus in some instances it is found that the on-off switch has been incorrectly placed in the neutral instead of the live wire so that the element remains live even when the switch is in the off position.

Under these conditions the unfortunate victim may touch the element and accidentally complete the circuit with his own body.

Almost all domestic supply is now by means of alternating current (A.C.) and in many instances the period of contact is prolonged by the muscles being thrown into tetanic spasm by the current so that the victim is unable to let go until the current is switched off, although some determined patients retain sufficient presence of mind to prise off the offending apparatus with their feet or wrench it away bodily until the plug comes out of its socket.

Direct current (D.C.) injuries may rarely be seen and in these there is a single sharp shock which causes a typical electrical burn and has the effect of blowing the victim off the source of supply. These injuries are sometimes sustained (usually by errant children) on the electric railway line (66o volts D.C.). Only the Southern Region of British Railways is extensively electrified, and although Dale of East Grinstead reported several of these injuries they are rarely seen in other parts of the country (Dale, I954).

The types of accidents described are all responsible for causing true electrical burns with all their typical features but it must be remembered that not all burns sustained on electrical apparatus are in fact of this nature; indeed, the majority of burns sustained by children when they fall and grasp the unguarded hot element of an electric fire are in fact heat burns and not electrical burns, as no current passes through the child. This has an important bearing on treatment and prognosis for these hot element burns are usually less deep than electrical burns (the damage is seldom deeper than the skin itself) and they are much more easily treated.

Superficial burns may also be caused by an electric flash, as when a fuse blows or an electric arc is struck as a result of a short circuit. These flash burns are again heat burns caused by a high temperature for a very short time and may present an alarming appearance as the face and hands may be blackened by a film of carbon. This is easily cleaned off and the underlying skin is found to be minimally damaged.

\section{Pathology}

There is still some disagreement as to the immediate cause of the damage, it being held on the one hand that it is due to heat generated by the passage of the current, and on the other to a specific action of the current on the tissues. It is true that high temperatures may be produced by the passage of electrical currents through tissues, but the natural history of electrical burns differs so clearly from that of thermal burns that it is impossible to avoid the conclusion that some specific effect is at least partially responsible for the damage.

The skin wound of an electrical burn is clearly circumscribed to the area of contact and this skin is dead. Deep to the skin the area of injury extends towards the other point of contact with destruction of deeper tissues. Because of their fluid content main vessels form good conductors and thrombosis of digital or other main vessels may occur with important consequences.

In a thermal burn (e.g. the pure heat burn from a hot element) the dividing line between destroyed and surviving tissue is clear cut and a healthy zone of reaction soon forms. The slough soon separates in Io to 14 days, exposing red healthy granulations on which skin grafts take readily, and it is exceptional for nerves, tendons or joints to be damaged or exposed during the course of healing.

In an electrical burn, however, the local defence reaction in the marginal zone of surviving tissue is imperfect and slow to develop; sloughs are slow to separate and the granulations, when they are exposed, are pale and oedematous, and free skin grafts take poorly. Furthermore, this surviving but damaged tissue, by reason of its depressed cellular activity, offers poor resistance to bacterial invasion and infection may lead to further necrosis of tissue with exposure of tendons or 



FIG. 2.- An electrical burn of the thumb treated by excision and the application of a cross finger flap from the dorsum of the index finger. (a) The narrow red margin round the dead white area is well shown in the smaller of the two areas. These are both 'entry' wounds. The exit wound is on the dorsum of the hand and is not shown. (b) Shows the flap in position. (c) Shows the donor area on the dorsum of the index finger covered by a Thiersch graft-at the first dressing. (d) Shows the final result. A patient of Mr. R. L. G. Dawson, F.R.C.S. 
joints which previously were thought to be out of danger.

\section{Clinical Features}

The injured area is often covered by a loose desquamated horny layer which has to be removed before the extent of the damage is disclosed. The area of skin destruction is then seen as a dead white area with a surrounding narrow rim of bright red (Fig. 1a). In the absence of infection the necrosed skin becomes black and separates slowly in many weeks leaving pale lilac-coloured granulations. Final healing is slow, but if the area of skin destruction is small the resulting scar is often surprisingly supple and satisfactory. When a wider area of the skin is involved contracture will occur unless damaged skin is replaced by grafting.

In more severe burns separation of the slough may result in the exposure of tendons, joints or bones, and if these are not protected by viable flaps bearing their own blood supply, sloughing of tendons and sequestration of bone and cartilage will follow.

In domestic supply accidents, although the burn may be deep, the area of deep damage corresponds in lateral extent approximately with the area of skin damage and deep prolongation under intact skin is limited. Occasionally main vessels underlying or close to the burn are seriously damaged and progressive distal thrombosis may result in gangrene of a digit or rarely the distal part of a limb not itself actually burned. With this exception the area of skin destruction remains confined to its original clearly defined limits and does not extend.

In high tension injuries the current may pass in through the skin of the hand and up the centre of the arm as it would up the core of a conducting cable, and widespread necrosis of forearm muscles, vessels and nerves may be present under undamaged skin. The current in these instances often finds its exit through the feet which may suffer comparable damage.

\section{General Effects}

If unconsciousness occurs in domestic accidents it is usually short-lasting and spontaneous recovery soon occurs. If contacts are exceptionally good, and also in high tension and lightning injuries, unconsciousness is common, may be prolonged, and is frequently accompanied by paralysis of respiration, the heart continuing to function. If artificial respiration can be started promptly, recovery from this state is possible (Arden et al., 1956).

In some patients cardiac arrest or fibrillation may occur as well as respiratory arrest, and although Jellinek has revived experimental animals by means of an electrical defibrillator, no recoveries from this condition in man have been recorded (Jellinek, 1952).

\section{Treatment}

\section{First Aid Treatment of Unconsciousness}

Artificial respiration should be started at once and continued - as in cases of drowning - until recovery occurs or unequivocable signs of death are present.

\section{Shock}

Surgical shock occurs only in high tension injuries with extensive tissue damage. In these cases red cell as well as plasma loss occurs and whole blood transfusions rather than plasma are indicated.

\section{Hot Element Burns}

As has been said these are heat burns and behave as such. They may be treated by the usual conservative methods till the sloughs have separated when thin split skin grafts should be applied. Later reconstructive work is frequently necessary as these grafts contract. Alternatively, when the area of full thickness loss is definite and circumscribed these burns are suitable for treatment by early excision and application of thick split skin or Wolfe grafts and under favourable circumstances this primary operation may provide a satisfactory definitive repair.

\section{True Electrical Burns-Domestic Power Supply Injuries}

Since these burns almost always involve the hand, the following remarks will apply particularly to injuries of this member, but the general argument and principles of treatment apply equally to other parts of the body.

Minor burns of restricted area may be allowed to heal spontaneously, but it is wise to warn the patient that healing will be slow and may take many weeks.

Treatment of more extensive burns is often difficult and there is no general agreement as to the best method to adopt. A brief review of the possibilities and arguments is therefore given.

\section{Early Excision and Grafting}

The burn is excised down to bleeding and apparently healthy tissue, and a free graft is applied. In some instances good results are obtained, but in others the graft fails to take, often tissue thought to be healthy becomes necrotic and not infrequently joints and tendons become exposed. Thus in ten out of twenty burns treated at Mount Vernon Hospital by this method the grafts failed to take, and in seven of the ten failures, joints or tendons were exposed and repair by flap 
or amputation became necessary. This has led some surgeons to advise late excision and grafting.

\section{Late Excision and Grafting}

This is advised on the grounds that after io to I4 days it is easier to differentiate between viable and non-viable tissue, that further necrosis will not occur and that grafts will take more readily. Examination of the records, however, gives no support to this hypothesis, for loss of grafts and further necrosis has occurred even when excision has been delayed as long as 21 days.

\section{Allowing Spontaneous Separation}

This is excessively slow, taking four to six weeks or more, and during the whole time the danger of infection is present. Even when spontaneous separation has occurred the granulations are so unhealthy that free grafts will often not take, exposure of tendons or joints still occurs and repair by flap is often necessary even after the long delay.

\section{Excision and Repair by Flap.}

Results show that this is a very satisfactory method with few failures, and the flaps have taken well and given good cover when used at all periods after burning from the day of injury to the 2 Ist day. This method owes its success to the fact that a flap of skin and fat bearing its own blood supply can resist infection and can protect viable but damaged tissue from the baneful effects of infection-a protection which free grafts cannot provide.

Repair by flap has a number of practical disadvantages if distant flaps (e.g. cross arm or abdominal) have to be used. The necessary positioning and immobilisation of the hand may interfere with active movements and may make limitation of oedema by elevation impossible. Furthermore, the patient will usually have to remain in hospital until the flap is divided. For these reasons local flaps should be used whenever possible as these will permit immobilisation in the position of choice, and elevation.

Cross finger flaps are very suitable for burns of the palmar aspect of fingers and thenar flaps may be used for burns of finger pulps (Fig. I). On the dorsum of the hand rotation or transposed flaps are of value although the limits between safety and necrosis are narrow and the surgeon should not be too ambitious.

The palm of the hand presents greater difficulty. The inelasticity of the skin makes true rotation flaps unsuitable and transposed flaps should be used with the greatest discretion.

For extensive lesions direct flaps from a distance become necessary. The opposite arm is usually a better site than the abdomen for it frequently allows better positioning of the fingers which very easily become stiff in extension if the hand is fixed to the abdomen.

As a rule every attempt should be made to preserve and repair fingers which promise to retain or regain good function, but if tendons or joints are obviously seriously damaged then early rather than late amputation should be considered. In this connection it should be remembered that it is frequently possible to retain a useful amount of the skin of an otherwise useless finger, and that this may make an excellent flap for repair of the palm or adjacent finger.

If tendons or joints become exposed during the course of surgical excision or following the failure of a free graft, then repair by flap becomes urgent if these structures are to be saved.

\section{.Summary of Surgical Treatment}

It is clear that the aim of treatment is to achieve intact skin cover at the earliest moment, to prevent infection and to allow early mobilisation. This will be achieved by:

I. Surgical excision of burn wound back to healthy tissue. This may involve amputation.

2. Repair by local flap if possible (filleted finger if available), otherwise distant flap.

3. Repair by free graft only if it is certain that excision is well clear of any possibly damaged tissue and if no important structures are likely to be exposed.

4. Elevation of the limb to minimise oedema.

5. Immobilisation with finger joints in flexion during the early stages of healing.

6. Active exercises of uninjured parts, and of injured parts as soon as they are healed.

\section{High Tension Injuries}

It will be found that most burns caused by the domestic power supply can be managed satisfactorily on the lines described, but high tension injuries are very much more difficult to manage and present some of the most difficult problems in reconstructive surgery. Usually two and sometimes three or four limbs are severely damaged and in addition the head and trunk may be involved. The basic nature and behaviour of the local lesions is the same as in the less severe domestic injuries but the destruction is so extensive that it is often impossible to employ the most desirable methods of local treatment and less reliable methods have to be used.

In seven high tension injuries treated at Mount Vernon Hospital, amputations of limbs were necessary in two patients, and severe and crippling injuries of hands were present in two others.

\section{BIBLIOGRAPHY}

DALE, R. M. (1954), Brit. F. plast. Surg., 7, 44. JELLINEK, S. (1952), Wien. klin. Wschr., 64, 620. ARDEN, G. P., et al. (1956), Brit. F. plast. Surg., 1, 1450. 